Muro de la Investigación, 2021(1), enero-junio ISSN: 2523-2886

Doi: https://doi.org/10.17162/rmi.v6i1.1435

\title{
Un estudio sobre dependencia emocional y estrategias de afrontamiento en mujeres víctimas de violencia doméstica
}

\author{
A study on emotional dependence and coping strategies in women victims \\ of domestic violence
}

\author{
Maribel Neira García ${ }^{1 a}$, Raquel Marilú Mejía Vásquez², \\ Daniel Josué Farfán Rodriguez ${ }^{3}$, \\ Universidad Peruana Unión, Lima, Perú ${ }^{123}$
}

Recibido: 09 de junio de 2020

Aceptado: 10 de diciembre de 2020

\begin{abstract}
Resumen
La presente investigación tuvo con objetivo determinar la relación entre la dependencia emocional y las estrategias de afrontamiento en mujeres víctimas de la violencia de su pareja y habitantes de la comunidad de Huaycán. El diseño de la investigación fue no experimental de corte trasversal y de alcance correlacional. Los instrumentos que se utilizaron fueron el Cuestionario Dependencia Emocional (CDE) creado por Leary en 1997 y validado por Lemos y Londoño (2006), el Inventario de Estrategias de Afrontamiento (CSI), elaborado por Lazarus y Folkman en 1981, validado por Cano, Rodríguez y Gracia en 2007. La muestra estuvo conformada por 90 mujeres. Se encontró que la dependencia emocional no se relaciona con las estrategias de afrontamiento. Sin embargo, se encontró relación significativa entre dependencia emocional y las dimensiones reestructuración cognitiva (rho -,307 $\mathrm{p}<0.05$ ), expresión emocional $(\mathrm{rho}=, 270 \mathrm{p}>0.05)$ y pensamiento desiderativo (rho $=, 343 \mathrm{p}<0.05$ ). Se concluye que, a mayor dependencia emocional en las mujeres, menor será la capacidad de reestructurar o percibir que se está vivenciando un problema. Por otro lado, se encontró que a mayor dependencia emocional en la mujer, mayor serán las reacciones emocionales y las dificultades para cambiar los pensamientos que producen malestar.
\end{abstract}

Palabras clave: dependencia emocional, estrategias de afrontamiento, víctimas, violencia

\begin{abstract}
The objective of the present investigation was to determine the relationship between emotional dependence and coping strategies in women victims of the violence of their partner and inhabitants of the community of Huaycan, in Lima, Peru. The design of the research was nonexperimental, cross-sectional and correlational in scope. The instruments used were the Emotional Dependency Questionnaire (CDE) created by Leary in 1997 and validated by Lemos
\end{abstract}

${ }^{\mathrm{a}}$ Correspondencia al autor:

maribelneira@upeu.edu.pe, autor2 raquelmejía@upeu.edu.pe 
and Londoño (2006), the Coping Strategies Inventory (CSI), prepared by Lazarus and Folkman in 1981, validated by Cano, Rodríguez and Gracia in 2007. The sample consisted of 90 women. It was found that emotional dependence is not related to coping strategies. However, a significant relationship was found between emotional dependence and the cognitive restructuring dimensions (rho -, $307 \mathrm{p}<0.05$ ), emotional expression (rho =, $270 \mathrm{p}>0.05$ ) and desiderative thinking (rho $=, 343 \mathrm{p}<0.05$ ). It is concluded that, the greater the emotional dependence of women, the lower the capacity to restructure or perceive that a problem is being experienced. On the other hand, it was found that the more emotionally dependent on women, the greater the emotional reactions and the difficulties in changing the thoughts that produce discomfort.

Keywords: emotional dependence, coping strategies, victims, violence

\section{Introducción}

En la actualidad, se exhibe una problemática alarmante respecto al maltrato contra el género femenino, y se piensa que una de cada tres mujeres en el mundo ha sufrido algún tipo de violencia por su pareja o terceros, en algún momento de su vida (OMS, 2017; Mallma, 2014). Según Castelló (2005) la mayor parte de mujeres padece de dependencia emocional, siendo susceptibles de establecer relaciones de pareja de orden patológico y gravemente desequilibrado, tomando un rol subordinado e inquebrantable ante la pareja.

Por otro lado, De la Villa et al. (2017) muestra que el 73,1\% de las mujeres manifiesta un cuadro de dependencia emocional. En Panamá, Gonzáles y Leal (2016) encontraron que el $41,9 \%$ de damas presentan un alto nivel de dependencia y el $42,7 \%$ de ellas se mantienen en un nivel medio. En cuanto a la prevalencia de este fenómeno en el Perú, Espíritu (2013) determinó que en Chimbote el $82.2 \%$ de mujeres agredidas también evidenciaban un nivel excesivo de dependencia emocional.

Asimismo, estudios realizados en Chiclayo por Castillo (2017) evidencian que las víctimas de violencia presentan con frecuencia altos niveles de dependencia emocional. Además, Aliaga y Travesaño (2015) evaluaron a 50 pacientes femeninas con depresión de un hospital psiquiátrico en Lima a fin de comprobar la relación entre dependencia emocional y pensamientos automáticos, encontraron que estas variables se correlacionan significativamente, concluyendo que el nivel de dependencia emocional será mayor respecto a la frecuencia de estos pensamientos. 
Por otro lado, según el análisis de la Dirección de Salud de Lima Este (2008) el distrito de Ate Vitarte se encuentra dentro de los ocho distritos de Lima con una alta tasa de violencia, específicamente la zona de Huaycán, ya que el $88,6 \%$ de las mujeres de esa zona sufren de violencia doméstica y por ende dependencia emocional; asimismo los objetivos específicos sintonizan con las dimensiones de estrategias de afrontamiento, para la cual se pretende determinar la relación entre dependencia emocional y resolución de problemas, reestructuración cognitiva, apoyo social, expresión emocional, entre otros.

En este sentido, el presente estudio permitirá describir la relación entre dependencia emocional y estrategias de afrontamiento, a fin de reforzar el conocimiento científico, en relación a las variables de estudio, puesto que existen pocas investigaciones sobre dichas al respecto. Por ende, esta investigación facilitará información actualizada, ordenada y sistematizada para futuras investigación.

\section{Método}

El presente estudio es de diseño no experimental y de corte transversal. Además, es de alcance descriptivo correlacional, pues tiene como finalidad determinar la relación entre las variables (Hernández, Fernández, y Baptista, 2006). La muestra escogida fue de 90 participante del sexo femenino con edades entre 17 a 56 años, que hayan sido víctimas de violencia por su pareja, los cuestionarios se aplicaron en el distrito de Ate, en la Comisaria de Huaycán a mujeres que acudieron a denunciar a su pareja por algún tipo de maltrato. La muestra se seleccionó a través de un muestreo no probabilístico de tipo accidental porque la población no fue elegida por conveniencia, sino de forma casual.

En este caso, se evidencia que el $81.1 \%$ se encuentran entre las edades $17-45$, el $58.9 \%$ provienen de la sierra, asimismo con respecto al estado civil 51.1\% son convivientes, el 55.6\% tienen un grado de instrucción de secundaria y el 68.9 pertenecen de la religión católica (tabla 1).

\section{Tabla 1}

Datos sociodemográficos

\begin{tabular}{|c|c|c|c|}
\hline Variables & & $f$ & $\%$ \\
\hline Edad & $17-45$ & 73 & 81.1 \\
\hline & $46-56$ & 17 & 18.9 \\
\hline
\end{tabular}




\begin{tabular}{lccc} 
Procedencia & Costa & 28 & 31.1 \\
& Sierra & 53 & 58.9 \\
Estado civil & Selva & 9 & 10.0 \\
& Separada & 25 & 27.8 \\
Grado de instrucción & Casada & 19 & 21.1 \\
& Conviviente & 46 & 51.1 \\
& Primaria & 24 & 26.7 \\
\multirow{3}{*}{ Religión } & Secundaria & 50 & 55.6 \\
& Técnico & 12 & 13.3 \\
& Superior & 4 & 4.4 \\
& Católica & 62 & 68.9 \\
& Evangélica & 18 & 20.0 \\
& Adventista & 4 & 4.4 \\
\hline
\end{tabular}

El Cuestionario Dependencia Emocional (CDE), fue creado por Leary (1997) y validada por Lemos y Londoño (2006). Para la utilización del cuestionario en el presente trabajo, se crearon baremos para poder hacer la interpretación de los resultados; asimismo, utilizaron los siguientes análisis estadísticos; el Alfa de Cronbach, alcanzó la escala general de 0.922, lo que evidencia una alta fiabilidad del constructo. Para la validez se sometió al criterio de jueces, donde 4 psicólogos expertos aportaron en la validación del contenido del instrumento. Para medir las estrategias de afrontamiento, se empleó el Coping Strategies Inventory (CSI) creado por Folkman y Lazarus (1981). Este instrumento consistió en 109 ítems, y fue validado por Cano, Rodríguez y García (2007).

\section{Resultados}

\section{Niveles de dependencia emocional}

El 52.2\% de las participantes evaluadas se encuentran en un nivel medio de dependencia emocional, lo que indica que las mujeres tratan de encontrar respaldo y seguridad en sus parejas. $\mathrm{Al}$ analizar las dimensiones, se puede notar que todas presentan porcentajes en ese nivel, como es el caso de la ansiedad por separación (52.2\%). Por otro lado, en la expresión afectiva de pareja, el $24.4 \%$ se sitúa en el nivel alto, lo que evidencia su constante necesidad de expresiones de afecto por su pareja a fin de calmar su inseguridad. No obstante, se evidencia, que un $33.3 \%$ se ubican en el nivel bajo respecto a la búsqueda de atención (tabla 2). 
Tabla 2

Niveles de dependencia emocional en mujeres

\begin{tabular}{lcccccc}
\hline & \multicolumn{2}{c}{ Bajo } & \multicolumn{2}{c}{ Medio } & \multicolumn{2}{c}{ Alto } \\
\cline { 2 - 7 } & $\mathrm{N}$ & $\%$ & $\mathrm{n}$ & $\%$ & $\mathrm{n}$ & $\%$ \\
Dependencia emocional & 22 & $24.4 \%$ & 47 & 52.2 & 21 & $23.3 \%$ \\
Ansiedad de separación & 22 & $24.4 \%$ & 47 & 52.2 & 21 & $23.3 \%$ \\
Expresión afectiva de pareja & 24 & $26.7 \%$ & 44 & 48.9 & 22 & $24.4 \%$ \\
Modificación de planes & 27 & $30.0 \%$ & 43 & 47.8 & 20 & $22.2 \%$ \\
Miedo a la soledad & 25 & $27.8 \%$ & 45 & 50.0 & 20 & $22.2 \%$ \\
Expresión limite & 24 & $26.7 \%$ & 46 & 51.1 & 20 & $22.2 \%$ \\
\hline
\end{tabular}

\section{Nivel de estrategias de afrontamiento}

En la tabla 3 se infiere que las mujeres frente problemas, utilizan un moderado uso de sus estrategias. Un $24 \%$ de las mujeres desean que los problemas que tienen no les resulte estresante(pensamiento desiderativo), sin embargo, un $31.1 \%$ presenta bajos niveles de retirada social, lo que indica estas mujeres se enfrentan a las personas que le generan reacciones emocionales con el fin de disminuir el estrés.

Tabla 3

Niveles de estrategias de afrontamiento en mujeres

\begin{tabular}{lcccccc}
\hline & \multicolumn{2}{c}{ Bajo } & \multicolumn{2}{c}{ Medio } & \multicolumn{2}{c}{ Alto } \\
\cline { 2 - 7 } & $\mathrm{n}$ & $\%$ & $\mathrm{n}$ & $\%$ & $\mathrm{n}$ & $\%$ \\
\hline Resolución de conflictos & 27 & $30.0 \%$ & 48 & $53.3 \%$ & 15 & $16.7 \%$ \\
Reestructuración cognitiva & 23 & $25.6 \%$ & 48 & $53.3 \%$ & 19 & $21.1 \%$ \\
Apoyo Social & 25 & $27.8 \%$ & 44 & $48.9 \%$ & 21 & $23.3 \%$ \\
Expresión emocional & 25 & $27.8 \%$ & 47 & $52.2 \%$ & 18 & $20.0 \%$ \\
Evitación de problemas & 26 & $28.9 \%$ & 43 & $47.8 \%$ & 21 & $23.3 \%$ \\
Pensamiento desiderativo & 26 & $28.9 \%$ & 42 & $46.7 \%$ & 22 & $24.4 \%$ \\
Retirada social & 28 & $31.1 \%$ & 46 & $51.1 \%$ & 16 & $17.8 \%$ \\
Cognitiva autocritica & 25 & $27.8 \%$ & 47 & $52.2 \%$ & 18 & $20.0 \%$ \\
\hline
\end{tabular}

\section{Prueba de normalidad}

La tabla 4 presenta los resultados de la prueba de normalidad de ajuste de KolmogorovSmirnov (K-S), evidenciándose que las variables dependencia emocional y estrategias de afrontamiento no presentan una distribución normal, y el coeficiente alcanzado (K-S) es significativo $(\mathrm{p}<0.05)$. 


\section{Tabla 4}

Prueba de normalidad de ajuste a la curva normal aplicadas a las variables

\begin{tabular}{llcccc}
\hline Instrumentos & Variables & Media & D.E & K-S & P \\
\hline & Dependencia global & 84,94 & 24,914 &, 100 &, 028 \\
& Resolución de problemas & 12,89 & 4,651 &, 153 &, 000 \\
& Reestructuración cognitive & 10,76 & 4,460 &, 089 &, 074 \\
Dependencia & Apoyo social & 11,13 & 5,022 &, 094 &, 049 \\
Afrontamiento & Expresión emocional & 13,49 & 4,440 &, 112 &, 007 \\
& Evitación de problemas & 9,42 & 4,388 &, 099 &, 029 \\
& Pensamiento desiderativo & 15,00 & 4,314 &, 169 &, 000 \\
& Retirada social & 10,78 & 4,242 &, 089 &, 077 \\
& Cognitiva autocritica & 9,64 & 5,260 &, 140 &, 000 \\
\hline
\end{tabular}

\section{Correlaciones las variables}

En la tabla 5, se señala que entre dependencia emocional y la dimensión resolución de problemas no se halló correlación (rho = -,015 p> 0.05); de la misma manera se da con la dimensión apoyo social ( $r h o=-, 072 \mathrm{p}>0.05)$ y evitación de problemas $(r=-, 072 \mathrm{p}>0.05)$. Sin embargo, existe correlación negativa y altamente significativa entre la dependencia emocional y reestructuración cognitiva ( $r h o=-, 307 \mathrm{p}>0.05$ ), es decir que cuanto mayor sea la dependencia en las mujeres menor será la capacidad de reestructurar la perspectiva negativa del problema. De la misma manera, se halló correlación positiva y significativa en expresión emocional (rho= ,270 p> 0.05), entendiendo que, a mayor dependencia emocional se intensificarán las expresiones emocionales.

\section{Tabla 5}

Correlaciones entre dependencia emocional y estrategias de afrontamiento

\begin{tabular}{lcc}
\hline Estrategias de afrontamiento & \multicolumn{2}{c}{ Dependencia emocional } \\
\cline { 2 - 3 } & Rho & $\mathrm{P}$ \\
\hline Resolución de problemas &,- 015 &, 885 \\
Reestructuración cognitive &,$- 307^{* *}$ &, 003 \\
Apoyo social &,- 072 &, 501 \\
Expresión emocional &, $270^{*}$ &, 010 \\
Evitación de problemas &,- 159 &, 134 \\
Pensamiento desiderativo &, $343^{* *}$ &, 001 \\
Retirada social &, 126 &, 238 \\
Cognitiva autocritica &, 148 &, 163 \\
\hline
\end{tabular}

$* \mathrm{p}<0.05 / * * \mathrm{p}<0.01$ 


\section{Discusión}

En cuanto a la relación entre dependencia emocional y la resolución de problemas, el coeficiente rho de Spearman indicó que no existe correlación (rho = -,015 p> 0.05), lo que significa que todas las parejas tienden a utilizar diferentes técnicas para resolver los problemas, no necesariamente siendo dependientes emocionalmente. Contrariamente, Martín (2016) halló correlación positiva entre dependencia emocional con las dimensiones resolución de conflictos, agresión psicológica y física leve ejercidas por la víctima; esto se daba por un patrón cultural que consiente a la agresión como respuesta adecuada a determinadas situaciones.

Por su parte, Niño y Abaunza (2015) muestran que las características propias del dependiente emocional presentan una alta impulsividad y agresión; puesto que las mujeres dependientes tratan de resolver sus conflictos, utilizando estrategias desadaptativas pero con una reducida capacidad cognitiva. No obstante, esto concuerda con Laca y Mejía (2017) donde no hallaron correlación entre las variables estudiadas; igual que Lancheros y Peñuela (2016), quienes señalan que las estrategias utilizadas por las mujeres con dependencia emocional no se dirigen a la solución de conflictos.

También se halló que existe correlación negativa y altamente significativa (rho) -,307 $p<0.05)$ entre la dependencia emocional y la reestructuración cognitiva; en otras palabras, las mujeres que toleran naturalmente los hechos estresantes, son incapaces de identificar la situación problemática que están viviendo. Ante esto, Sosa (2017) afirma el sentimiento de inferioridad que las mujeres dependientes muestran y que aceptan el maltrato de su pareja como algo normal. Agregado a esto, Castelló (2005) descubrió que estos patrones de conducta se formaron en la infancia, producto del maltrato parental, donde las conductas y agresiones de todo tipo fue algo cotidiano para ellas.

Asimismo, se encontró que no hay relación entre dependencia emocional y apoyo social $($ rho $=-, 072 \mathrm{p}>0.05)$. Esto se explica en que, las mujeres con estas características, podrán o no buscar ayuda o respaldo frente a los acontecimientos estresantes que vivencian. Según Castelló (2005) estas mujeres se aíslan voluntariamente de su entorno familiar o social a fin de someterse a su pareja y hacerlo propio, es decir se sienten desestimadas, humilladas y consideran a su

pareja como el centro de su existencia. En este sentido, Álvarez, (2017) explica que las mujeres con un contexto de agresión y conflicto manifiestan una marcada inclinación hacia un trastorno 
de adaptación.; además, atenúan sus relaciones sociales aferrándose solo a su pareja, y eso les impide ir a buscar ayuda (Ríos y Londoño, 2012).

Entre la relación de la dependencia emocional y expresión emocional, se encontró una correlación positiva y significativa ( $\mathrm{rho}=, 270 \mathrm{p}>0.05)$, a mayor dependencia emocional, mayor se incrementarán las reacciones emocionales. Esto coincide con Urbiola, et al. (2017) quienes afirman que el exceso de dependencia sería un factor desencadenante para un estado depresivo; asimismo Momeñe, Jáuregui y Estévez (2017) encontraron la correlación entre la dependencia emocional, la regulación emocional y el abuso psicológico; además consideran que el apego afecta a los procesos de regulación emocional, los cuales inciden en las relaciones.

En otro contexto, Jaller y Lemos (2009) aducen que los patrones desadaptativos tempranos se relacionan con la falta de manejo de sí mismos, la tolerancia a la frustración y en controlar sus impulsos en personas con dependencia emocional. De acuerdo a esto Llerena (2017) confirma que el apego ansioso desarrollado en la infancia, donde la figura materna estaba ausente para satisfacer las necesidades de forma plena, establece un vínculo inadecuado y negativo, convirtiéndose en un factor de riesgo para el aparecimiento de alteraciones psicológicas como: descontrol emocional, frustración, miedo a permanecer solo, desesperanza, desconfianza e inseguridad hacia el mundo que le rodea en la edad adulta.

Por otro lado, los resultados del presente estudio no afirman la correlación significativa entre dependencia emocional y la evitación de problemas ( $\mathrm{rho}=,-159 \mathrm{p}>0.05)$. Esto evidencia que las mujeres dependientes utilizan otros recursos para el manejo de problemas, porque estas han sufrido maltratos psicológicos y físico por su pareja y es difícil que eviten pensar en estos hechos. Por ello, Galego, Satibáñez e Iraurgi (2016) mencionan que las mujeres víctimas de violencia presentan un cuadro común de culpabilizar a otros; las personas con estas conductas tienen menos control sobre sus pensamientos intrusivos y también presentan pensamientos catastróficos, enfocándose en la situación conflictiva que han experimentado y produciendo en ellas un cuadro de estrés postraumático.

En otro estado de cosas, en la correlación de dependencia emocional y pensamiento desiderativo, se muestra una correlación positiva y altamente significativa (rho =,343 p<0.05) lo que señala que, a mayor dependencia emocional mayores serán las dificultades para cambiar los pensamientos conflictivos. Esto significa que las mujeres que se aferran e idealizan a sus parejas y presentan un miedo extremo a la separación, carecen de decisión para buscar solución 
a sus angustias. En este contexto, Sepúlveda, Romero y Jaramillo (2012) confirman que la toma de decisiones y la opinión que establezcan estas mujeres se basan en sentimientos e ideas de placer, un énfasis en las emociones, y no miden las consecuencias que puedan generar su sumisión hacia las manifestaciones agresivas de su pareja.

Contrario a este resultado, Rubiela y Rojas (2017) menciona diferentes razones por las cuales las mujeres que deciden denunciar a sus parejas por violencia, porque han pasado por el ciclo completo de la violencia conyugal, además por el cansancio de la situación violenta, el deseo de cambio por parte de la pareja para continuar con el hogar, el miedo a que la violencia se traslade a sus hijos o por el acto violento que la llevó a denunciar fue tan abrupto que le dio a entender que su vida corría peligro frente a otro encuentro donde se aplicara la violencia física. Asimismo Sierra, et al. (2014) agrega que estas mujeres, al momento de tomar consciencia de su sufrimiento y se atreven a denunciar enfrentando los obstáculos a la hora de buscar ayuda. Estos resultados, dan a entender que las mujeres que permanecen en esta relación tienen pensamientos distorsionados, del cambio de comportamiento de su pareja, sin embargo, cuando perciben que la conducta de maltrato es extrema, tienen miedo que se repita y buscan ayuda.

Cabe mencionar que no existe correlación significativa entre la dependencia emocional y la dimensión retirada social $(\mathrm{rho}=, 126 \mathrm{p}>0.05)$ es decir que las mujeres dependientes emocionalmente no siempre tienden a aislarse de la persona que le produce tensión o frustración. Frente a ello, Bradshaw, menciona que la falta de identidad propia conlleva a perder la conexión consigo mismo, con lo que siente, necesita, y desea; así mismo piensa que su valor como persona depende de la opinión de los demás, hasta el punto de darle más importancia a otros que a sí mismo (citado por Montalvo, et al., 2011). Por su parte, Amor, Bohórques y Echeburúa (2006) manifiestan que hay diversas circunstancias que explican la permanencia de la mujer maltratada en convivencia con el agresor, primero están las contextuales, donde influyen la dependencia económica, presiones sociales y familiares; segundo están las personales, como es las creencias, sentimientos de baja autoestima, malestar emocional y miedo al extremo agresor y tercero están las vinculadas a los maltratadores donde existe la dependencia emocional a la víctima, arrepentimientos intermitentes por su comportamiento violentos, amenazas de muerte a la mujer o hijos.

Escudero, et al. (2005) manifiestan que la violencia de género se produce por las estrategias de persuasión coercitiva del maltratador sobre la víctima. Estas estrategias generan 
un progresivo estado de confusión de emociones, distorsión de pensamientos y paralización que dificultan que la mujer abandone la relación establecida por el sujeto maltratador, de esta manera logra aislar a la víctima de todos los soportes sociales debilitando su habilidad para resistir. Además Montero (2001) señala algunas teorías que explica los vínculos paradójicos en las relaciones íntimas de la violencia, considera el desequilibrio de poder y la intermitencia en el tratamiento bueno y malo, generan en la mujer maltratada el desarrollo de un vínculo traumático producido por las alternativas de refuerzos positivos y castigos, sin embargo esta teoría se basa en el condicionamiento instrumental donde algunos aspectos del repertorio de victimización principalmente aquellos referidos indefensión aprendida y al efecto sobre la víctima de los períodos de arrepentimiento del agresor; otra teoría que se ajusta al describir la dependencia emocional es el síndrome de Estocolmo, es un tipo de estado disociativo que lleva a la víctima a negar la parte violenta del comportamiento del agresor mientras forman un vínculo con el lado que percibe más positivo, ignorando sus propias necesidades.

Además, entre la dependencia emocional y la dimensión cognitiva autocritica (rho =, 148 p<,168) no se encontró correlación, es decir que las mujeres dependientes emocionalmente no siempre tienden a criticarse por circunstancias adversas. Sin embargo, este resultado es contrario a lo mencionado por Lemos, Londoño y Zapata (2007) quien considera que los dependientes emocionales poseen un pensamiento distorsionado de falacia de control, en lo cual asumen la culpa de las situaciones emocionalmente dolorosas dentro de sus relaciones de pareja, incluso de aquellos que no son responsables. Esto se debe a que las mujeres que inicialmente se encuentran dentro del ciclo de violencia tienden a idealizar a su pareja defendiendo su mal comportamiento; en contraste, las mujeres que han salido de este ciclo y decidido denunciar comprender la situación conflictiva y tienden a culpabilizar a otras personas de lo sucedido es una forma de distrés emocional diferente al miedo o la vergüenza y que contribuye a la victimización; también se considera otra razón según este tipo de estrategia (culpabilizar) guarda una estrecha relación con el hecho de que las mujeres que participan de un estudio toman la decisión de actuar y de rebelarse contra la violencia de género (Gallego, Santibáñez e Iraurgi, 2016).

\section{Referencias}

Aliaga, D., \& Travesaño, J. (2015). Dependencia emocional y pensamientos automáticos en 
pacientes de sexo femenino con depresión de un hospital psiquiátrico de Lima Metropolitana (Tesis licenciatura). Universidad Peruana Unión, Lima, Perú. Recuperado de: Recuperado de: http://repositorio.upeu.edu.pe/bitstream/handle/UPEU/136/Diana_Tesis_bachiller_2016. pdf?

Álvarez, R. A. (2017). Relación entre dependencia emocional, autoestima y apoyo social con los trastornos de adaptación en personas separadas. Universidad de San Buenaventura Medellín, Facultad de Psicología. Relación. Recuperado de: https://www.google.com/search?

Amor, P., Bohórquez, I., \& Echeburúa, E. (2006). ¿Por qué ya qué coste físico y psicológico permanece la mujer junto a su pareja maltratadora? Acción psicológica, 4(2). Recuperado de: http://www.redalyc.org/articulo.oa?id=344030758006.

Cano, F., Rodríguez, L., \& García, J. (2007). Adaptación española del inventario de estrategias de afrontamiento. Revista Actas Especialista en Psiquiatría. 35(1), 29-39. Recuperado de: https://personal.us.es/fjcano/drupal/files/AEDP\%2007\%20(esp).pdf.

Castelló, J. (2005). Dependencia emocional: Características y tratamiento. España: Alianza.

Castillo, E. G. (2017). Dependencia emocional, estrategias de afrontamiento al estrés y depresión en mujeres víctimas de violencia de pareja de la ciudad de Chiclayo. Universidad San Martín de Porres. from www.repositorioacademico.usmp.edu.pe/handle/usmp/2656

Espíritu, L.C. (2013). Dependencia emocional en mujeres violentadas y no violentadas de Nuevo Chimbote. Revista de Investigación de estudiantes de Psicología "JANG" de la Universidad Cesar Vallejo, (2), 123-140. Recuperado

Martín, P. (2016). Sexismo, dependencia emocional y resolución de conflictos en parejas adolescentes. Universidad de Granada.

Galeano, R. L. (2017). Bienestar psicológico y estrategias de afrontamiento en funcionarios de una Institución Educativa. Revista de Investigacion En Ciencias Socailes Y Humanidades, 4(2), 31-38.recupeardo de: Recuperado de revistacientifica.uamericana.edu.py/index.php/academo/article/view/227

Galego, V., Satibáñez, R., \& Iraurgi, I. (2016). Estrategias cognitivas de regulación emocional en mujeres de situación de maltrato. Pedagogia Social Revista Interuniversitaria, (28), 115-125. Retrieved

from http://www.redalyc.org/articulo.oa?id=135047100010\%0ACómo

Gonzáles, H., \& Leal, R. (2016). Dependencia Emocional Como Factor De Riesgo Para La Violencia De Pareja En Mujeres Del Sector Paraíso- Corregimiento Mateo Iturralde Distrito De San Miguelito - Panamá . Emotional Dependency As a Risk Factor for Intimate Partner Violence in Women Del Se. Tendencias En Psicología, 1(2), 25-35. Recuperado de: revistas.upagu.edu.pe/index.php/TP/article/download/332/256

Hernández, R., Fernández, C., \& Baptista, P. (2006). Analisis de los datos cuantitativos. Metodología de la investigación (McGraw-Hil). México. https://doi.org/10.6018/turismo.36.231041 
Laca, F., \& Mejía, C. (2017). Dependencia emocional, consciencia del presente y estilos de comunicación en situaciones de conflicto con la pareja. Enseñanza E Investigación En Psicología, 22(1), 66-75. Recupeado de: http://www.redalyc.org/html/292/29251161006/

Lancheros, G., \& Peñuela, G. P. (2016). Estrategias de afrontamiento en mujeres víctimas de violencia de pareja en la ciudad de bogotá (Tesis de licencitura). Fundación universitaria los libertadores. Bogotá. Recuperado de repository.libertadores.edu.co/handle/11371/1390

Llerena, S. (2017). Creencias Irracionales y Dependencia Emocional. Universidad técnica de ambato facultad de ciencias de la salud carrera de psicología clínica. Retrieved from http://repositorio.uta.edu.ec/jspui/handle/123456789/24761\}

Lemos, M., \& Londoño, N.H. (2006). Construcción y validación del cuestionario de dependencia emocional en población colombiana. Revista Acta Colombiana de Psicología, 9(2),127-140. Recuperado de http://www.redalyc.org/pdf/798/79890212.pdf.

Mallma, N. (2014). Relaciones intrafamiliares y dependencia emocional en estudiantes de psicología de un Centro de Formación Superior de Lima Sur (Tesis para obtener el título de licenciatura). Universidad Autónoma. Perú. Recuperado de: http://repositorio.autonoma.edu.pe/bitstream/autonoma/96/1/mallma\%20fernandez.pdf.

Martín, P. (2016). Sexismo, dependencia emocional y resolución de conflictos en parejas adolescentes. Universidad de Granada.

Organizacion Mundial de la salud. (2017). Violencia contra la mujer.

María de la Villla, C. S., Blanco, P., Palacios, L., Cruz, R., García, R., Delgado, R., ... L., Q. (2017). Interdependencia afectiva en dependencia sentimentales, (February), 121.Recuperado de: https://www.researchgate.net/publication/313250939

Martín, P. (2016). Sexismo, dependencia emocional y resolución de conflictos en parejas adolescentes. Universidad de Granada.

Miracco, M. C., Rutsztein, G., Lievendag, L., Arana, F. G., Scappatura, M. L., Elizathe, L. \& Keegan, E. (2010). Estrategias De Afrontamiento En Mujeres Maltratadas: 5967.recuperado

de: https://www.google.com/search?q=ESTRATEGIAS+DE+AFRONTAMIENT

Momeñe, J., Jáuregui, P., \& Estéz, A. (2017). El papel predictor del abuso

psicológico y la regulación emocional en la dependencia emocional., 25, 65-78

Montalvo, J., Nápoles, O., Espinosa, M., \& González, S. (2011). Efectos Cualitativos de una Técnica de PNL en la Dependencia Emocional de Pareja. Revista Electrónica de Psicología Iztacala, 14(1), 1-23. Retrieved from http://www.revistas.unam.mx/index.php/repi/article/view/24784/23256

Montero, A. (2001). Síndrome de adaptación paradójica a la violencia doméstica: una propuesta teórica. Red de Revistas Científicas de América Latina, El Caribe, España Y Portugal, 12(1), 5-31. Retrieved from http://www.redalyc.org/articulo.oa?id=180618320001\%0ACómo

Pereira, T. R. \& Lost, S. C. (2012). Relación entre apoyo social y capacidad funcional de los mayores con los cambios cognitivos, 20(4), 1-8.recuperado de:https://www.google.com/search?q=Relación+entre+apoyo+social+y+ca 
Polo, C., López, M., Olivares, D., Escudero, A., Rodríguez, B., \& Fernández, A. (2006). Autoinculpación en mujeres que sufren maltrato por parte de su pareja. Revista asociación española Neuropsiquiatría, 26(1), 71-86. Recuperado de: scielo.isciii.es/scielo.php?script=sci_arttext\&pid=S0211-57352006000100005

Sepúlveda, A. C., Romero, A. L., \& Jaramillo, L. (2012). Estrategias de afrontamiento y su relación con depresión y ansiedad en residentes de pediatría en un hospital de tercer nivel. Bol Med Hosp Infant Mex, 69(5), 347-354. recuperado de: https://www.google.com/search?client=firefox-b-

ab\&ei=H6BnWoPEOMG0zwLt246ADQ\&q=Bienestar+psicológico+y+estrategias+de+a frontamiento+en+funcionarios+d+eua+institucion+educativa $\&$.

Sierra, J. C., Paz, M. Buela, G., Salinas, J. M., \& Monge, F. (2014). Variables asociadas a la experiencia de abuso en la pareja y su denuncia en una muestra de mujeres. Universitas Psychologica, 13(1), 37-46. recuperado de: https://doi.org/10.11144/Javeriana.UPSY131.

Sosa, D. B. (2017). Dependencia emocional y autoestima en mujeres víctimas de violencia conyugal con y sin antecedentes de maltrato infantil - San Juan de Lurigancho. Lima, 2017”. Facultad de Humanidades. Universidad César Vallejo. Recuperado de: https://www.google.com/search?q=Dependencia+emocional+y+autoestim

Rubiela, M., \& Rojas, M. (2017). Motivos, temores y expectativas que surgen frente a la denuncia en mujeres denunciantes por maltrato conyugal atendidas en una Comisaria de Familia del Valle de Aburrá de enero a noviembre de 2016. Corporación Universitaria Minuto de Dios. 